\title{
Über die Koeffizienten der Reizbildung im Herzen.
}

\author{
Von \\ Prof. Dr. H. E. Hering, Köln-Lindenthal.
}

(Eingegangen am 13. Dezember 1920.)

Im Jahre 1911 schrieb ich ${ }^{1}$ ) auf S. 508 der unten zitierten Abhandlung: „Als Ursprungsreiz habe ich ${ }^{2}$ ) seinerzeit (1902) jenes Geschehen bezeichnet, das dort vor sich geht, wo die automatische Herztätigkeit ihren Ausgangspunkt nimmt. Dazu sei bemerkt, daß der Ausdruck ,Reiz ${ }^{6}$ von mir nur beibehalten worden ist, weil er üblich ist; er würde aber besser durch einen anderen Ausdruck ersetzt werden; denn unter Reiz verstehen wir gewöhnlich nur eine Bedingung, unter der etwas geschieht, während beim Herzen mehrere Bedingungen erforderlich sind, damit es z. B. wieder zu schlagen anfängt, und gewiß niemand gerade jene Bedingung als den Ursprungsreiz wird bezeichnen wollen, die bei Vorhandensein aller übrigen Bedingungen noch hinzukommen muß, damit das Herz zu schlagen anfängt. ,Die Minimalbedingungen', sagte ich ${ }^{3}$ ) 1905 auf S. 128, ,unter denen ein schlagloses Säugetierherz wieder zu schlagen anfängt, sind enthalten in einer entsprechend warmen, etwas Sauerstoff absorbiert enthaltenden, alkalischen Kochsalzlösung. Lassen wir den Sauerstoff oder die Wärme oder die Salze usw. weg, dann schlägt es nicht. Ist nun der Sauerstoff oder die Wärme usw. als Ursprungsreiz zu bezeichnen? Gewiß nicht. Aber auch die Minimalbedingungen können unter Umständen vorhanden sein, ohne daß das Herz schlägt. Fügen wir jedoch noch eine Bedingung hinzu, d. h. erregen wir den Accelerans, oder fügen wir zu der Durchströmungsflüssigkeit Adrenalin oder Calcium, dann kann das Herz wieder zu schlagen anfangen."

Ich hätte nun nicht erwartet, daß heute noch Forscher ${ }^{4}$ ) eine Mitteilung unter dem Titel veröffentlichen würden: „Untersuchungen über die Ursache des Herzschlages"c und in dieser Mitteilung anführen, „daß die Kohlensäure nicht eine Bedingung, sondern offenbar selbst der Reiz für die Herztätigkeit ist". Ich hätte es nicht erwartet, weil ich glaubte, daß die ätiologische Denkweise infolge der verschiedenen Bestrebungen, sie in die richtigen Bahnen zu leiten, jetzt endlich eine den geklärten Verhältnissen adäquate sein würde. Dies trifft nun nicht $\mathrm{zu}$, vielmehr widerlegt diese Mitteilung neuerdings die Behauptung jener, die meinen, daß die Plu ra l it ät der Ursachen eine „Binsenwahrheit"sei.

1) Arch. f. d. ges. Physiol. 141.

2) Arch. f. d. ges. Physiol. 92, 392.

3) Physiol. Centralbl. 19, H. 15. 1905.

4) G. Mansfeld und A. v. Szent-Györgyi, Arch.f. d. ges. Physiol. 184, 236. 
Wollte man die Frage stellen, ob die Kohlensäure die Ursache des Herzschlages sei, so wäre diese Fragestellung unzutreffend. Gleichgültig, ob man als Bezeichnung den Ausdruck Ursache, Bedingung oder Koeffizient, wie ich ${ }^{1}$ ) es seit 1912 tue, verwendet, die Frage hätte nur zu lauten, ob die Kohlensäure eine Ursache, eine Bedingung oder ein Koeffizient der Reizbildung sei. Dementsprechend ist es auch nicht zutreffend, „daß die Kohlensäure offenbar selbst der Reiz für die Herztätigkeit ist", oder daß die Autoren ,,in der Kohlensäure den inneren Herzreiz erkannt haben", sondern die $\mathrm{CO}_{2}$ ist nur einer der vielen Koeffizienten, welche die Reizbildung bewirken. Dem Ursprungsreiz entspricht in Wirklichkeit ein Geschehen, an dem sich eine Mehrzahl von Koeffizienten beteiligen, von denen einer die Kohlensäure ist. So viel zur ätiologischen Denkweise und der sich daraus ergebenden Folgerung, daß G. Mansfeld und A. v. Szent - Györgyi nicht etwa in der Kohlensäure „die Ursache des Herzschlages" gefunden haben.

Was die Annahme anbelangt, daß die Kohlensäure ein Koeffizient der Reizbildung sei, so sei bemerkt, daß schon E. G. Martin ${ }^{2}$ ) im Jahre 1906 auf Grund von Versuchen an Kaltblüterherzen annahm, daß die Kohlensäure „den Reiz" für die Herzbewegung darstelle. Während ein Herzkammerstreifen in reiner Kochsalzlösung nicht schlug, begann er sofort zu schlagen, wenn zu der Kochsalzlösung Kohlensäure hinzugefügt wurde. Damit aber die Kohlensäure ihre Wirkung entfalten könne, müßten nach Martin Calciumionen in diffusibler Form vorhanden sein. Das geschehe dadurch, daß das Kochsalz durch Massenwirkung die Kalkverbindungen des Herzens diffusibel mache.

$\mathrm{Ich}^{3}$ ) habe die Kohlensäure als Koeffizient für die heterotope Reizbildung angesehen: 1. weil bei Erstickung der Säugetiere Vorhöfe oder Kammern leicht ins Flimmern geraten, 2. nach Verschluß einer Coronararterie es in dem von ihr besorgten Bezirk vor dem Auftreten der heterotopen Reizbildung und des Flimmerns zu einer lokalen Erstickung kommt, 3. weil Magnus beobachtet hat, daß die Durchströmung der Coronargefäße mit Kohlensäure die Kammern des isolierten Säugetierherzens zum Flimmern bringt und 4. weil das Flimmern den höchsten Grad heterotoper Reizbildung darstellt. Ich nehme nun schon seit längerer Zeit an, da $B$ die heterotopen Ursprungsreize ihrer Natur nach den nomotopen Ursprungsreizen gleichartig sind, was ich ${ }^{4}$ ) als Ho mot y pie der Reizbildung bezeichnet habe. Hiermit stimmt überein, daß die Kohlensäure wie für die heterotope Reizbildung, so auch für die nomotope ein Koeffizient ist.

1) Die Koeffizientenlehre (Pluralität der Ursachen). Die Naturwissenschaften 1913, H. 7; Münch. med. Wochenschr. 1919, Nr. 19.

2) Amer. journ. of physiol. 1906, H, 15.

3) Arch. f. d. ges. Physiol. 163, 22.

4) Kölner Festschrift, Bonn 1915, Marcus \& Webers Verlag. 
Bezüglich der Ansicht von Langendorff ${ }^{1}$ ): „,Das Lebensprodukt der Zelle ist ihr Erreger", sei folgendes bemerkt. Wir pflegen einen Ruhestoffwechsel (RS) und $\epsilon$ inen Tätigkeitsstoffwechsєl (TS) zu unterscheiden. Wenn während der Tätigkeit des Muskels sich Kohlensäure bildet, so hat diese beim TS sich bildende Kohlensäure die Muskeltätigkeit nicht ausgelöst, da diese Kohlensäure erst nach der Auslösung der Muskeltätigkeit auftritt. Solche während der Muskeltätigkeit gebildete Kohlensäure kann aber sehr wohl als Hormon, ähnlich vielen anderen Hormonen, die Tätigkeit anderer Zellen beeinflussen, so auch jener Zellen, von denen die Atmung, oder jener, von denen jeweils die Herztätigkeit ausgeht. Wir wissen heute, daß auch beim RS Kohlensäure gebildet wird (Fle tcher, Thunberg), und es wäre vielleicht möglich, daß die $\mathrm{RS}-\mathrm{CO}_{2}$ ein Koeffizient für die Reizbildung in der Medulla oblongata oder im Herzen sein könnte, also daß nach Langendorff „die Reize an Ort und Stelle als Produkt ihrer vitalen Dissimilation entstehen". Es fragt sich jedoch, ob die beim RS nor maler weise gebildete Menge $\mathrm{CO}_{2}$, ja auch ob die während des TS der Reizbildungszellen lediglich von diesen an Ort und Stelle gebildete $\mathrm{CO}_{2}$ hinreicht, um als Koeffizient für die Reizbildung zu genügen; das muß doch noch dahingestellt bleiben. Insofern muß auch die Richtigkeit des Satzes von Lan ge nd or ff ",das Lebensprodukt der Zelle ist ihr Erreger", so wie er ihn verstanden hat, noch dahingestellt bleiben, während ich meine, daß es nicht zweifelhaft ist, daß die Dissimilationsprodukte der Zellen Koeffizienten der Erregung anderer Zellen sein können und dementsprechend die von a nderen Zellen gebildete $\mathrm{CO}_{2}$ ein Koeffizient der Reizbildung im Herzen wie in der Medulla oblonga ta ist. Vielleicht wirkt die von den Reizbildungsstellen an Ort und Stelle gebildete $\mathrm{CO}_{2}$ und die von den anderen Zellen des Körpers gebildete $\mathrm{CO}_{2}$ zusammen als Koeffizient der Reizbildung.

In den Versuchen von M. u. S. wurde sowohl die Zufuhr der $\mathrm{CO}_{2}$ zu verhindern, als auch die gebildete $\mathrm{CO}_{2}$ zu binden gesucht, also sowohl die Wirkung der ortsfremden, zugefïhrten wie auch die der an Ort und Stelle gebildeten ortseigenen $\mathrm{CO}_{2}$ auszuschalten versucht. In den Versuchen am Säugetierherzen war den Autoren unverständlich, wie in den Reizbildungsapparaten am venösen Herzende ,eine wirksame Konzentration an $\mathrm{CO}_{2}$ entstehen könnte“, wenn nicht ,trotz Coronarkreislauf auch noch eine direkte Stoffaufnahme von den Herzhöhlen erfolgt, $d a ß$ also die Apparate der normalen Reizbildung auch hier unter derHerrschaft des venösen Blutes stehen ". Diese Auffassung widerspricht aber direkt der von Langendorff, daß die ,,an Ort und Stelle" gebildeten Stoffwechselprodukte die Zellerreger seien.

Was die Versuche anbelangt, um zu prüfen, ,ob ein Stoffaustausch zwischen den Zellen des Herzens und dem Inhalt der Herzhöhlen am

1) Ergebn. d. Physiol. 1902, S. 324. 
Warmblüter durch einfache Diffusion erfolgen kann", so sei hierzu folgendes bemerkt. Zunächst, daß es lange bekannt ist, daß Säugetierherzen bei lediglicher Speisung von den Herzhöhlen aus regelmäßig schlagen können. Damit hat sich besonders F. H. Pratt ${ }^{1}$ ) im Jahre 1898 beschäftigt. Ich habe diese Speisung wiederholt mit einfacher Ringerlösung ausgeführt, während Pratt seinerzeit Blut zu seinen Versuchen nahm. Dabei wird das Herz durch die Thebesischen Gefäße bzw. auch durch die Coronarvene rückläufig gespeist. Dies ist M. u.S. allem Anschein nach nicht bekannt gewesen, da sie von einer „einfachen Diffusion“ sprechen. Wenn, nachdem die Durchströmung der Coronargefäße mit einer $\mathrm{CO}_{2}$-freien Lösung eine heterotope Reizbildung bewirkt hatte, die Durchströmung der Herzhöhlen mit einer $\mathrm{CO}_{2}$-haltigen Lösung die nomotope Reizbildung wiederhergestellt hat, so geht daraus jedesfalls nicht hervor, daß die Aufnahme von $\mathrm{CO}_{2}$ von den Herzhöhlen aus durch Diffusion erfolgt. Es beweist aber auch nicht die Annahme der Autoren, daß die Reizbildung im Sinusknoten ,unter der Herrschaft des venösen Blutes stehe". Es sei ferner darauf hingewiesen, daß normalerweise der Säftestrom auf den Wegen, auf denen es möglich ist, das Herz von den Herzhöhlen aus zu speisen und zum regelmäßigen Schlagen zu bringen, in der Richtung gegen die Herzhöhlen erfolgt, also in gerade umgekehrter Richtung als in den obengenannten Versuchen. $\mathrm{Ob}$ normalerweise eine Diffusion von $\mathrm{CO}_{2}$ aus den Herzhöhlen des Warmblüters in das Herz hinein erfolgt, muß also vorläufig dahingestellt bleiben. Man sollte doch wohl annehmen, daß, wenn eine Diffusion von $\mathrm{CO}_{2}$ aus der Herzhöhle in das Gewebe des Säugetierherzens hinein erfolgt, die $\mathrm{CO}_{2}$-Spannung außerhalb größer sein müßte als in den Geweben. Wie groß die $\mathrm{CO}_{2}$-Spannung der Flüssigkeit war, welche in jenen Versuchen die Herzhöhle speiste, geht aus den Angaben der Autoren nicht hervor. $\mathrm{Da} ß$ sie aber nicht so hoch zu sein braucht wie in jenen Versuchen, das geht aus meinen obenerwähnten Versuchen hervor, in denen schon die Speisung mit gewöhnlicher Ringerlösung (also ohne erhöhten $\mathrm{CO}_{2}$-Gehalt) genügte, um von den Herzhöhlen aus das Säugetierherz schlagen zu lassen.

Endlich sei noch bemerkt, da $B$ ich noch nicht geneigt bin, ,,den Beweis“" dafür erbracht zu sehen, ,daß die erregende Wirkung der $\mathrm{CO}_{2}$ auf die Apparate der Reizbildung keine Wirkung der H-Ionen ist, sondern nur eine spezifische Kohlensäurewirkung sein kann". Dazu bedarf es noch weiterer Untersuchungen. Man vergleiche hierzu nur die Arbeiten über die chemische Regulation der Atmung durch die Wasserstoffionenkonzentration, deren Literatur von den Autoren nur bis zum Jahre 1911 erwähnt wird.

1) Amer. journ. of physiol. 1898, S. 86.

Anm.: M. u. S. gebrauchen den Ausdruck Tawaraknoten, der für das Säugetierherz gilt, auch für das Froschherz, obwohl er für dieses nicht zutreffend ist. 